\title{
ANÁLISE ERGONÔMICA DO TRABALHO EM UM RESTAURANTE SITUADO NO INTERIOR DO RIO GRANDE DO SUL
}

\author{
Bruna Dalcin Pimenta ${ }^{l *}$, Airton dos Santos Alonço ${ }^{1}$, Tiago Rodrigo Francetto ${ }^{2}$, Jhosefe Bruning ${ }^{1}$, Miguel Chaiben Neto ${ }^{l}$, Silvana \\ Antunes Rodrigues ${ }^{1}$ \\ IPrograma de Pós-Graduação em Engenharia Agrícola, Universidade Federal de Santa Maria, CEP 97105-900, Santa Maria/RS, \\ Brasil. \\ 2Universidade Federal de Santa Maria, CEP 96506-310, Cachoeira do Sul/RS, Brasil.
}

*E-mail: $\underline{\text { brunadpimenta@gmail.com }}$

\section{RESUMO}

A ergonomia se diferencia de outras áreas de conhecimento pela sua característica interdisciplinar e sua natureza aplicada, isto é, a adaptação do posto de trabalho e ambiente às características e necessidades do trabalhador. O objetivo deste trabalho é avaliar o ambiente e a postura corporal de funcionários de um restaurante privado do interior do Rio Grande do Sul para indicar possíveis melhorias tanto para o estabelecimento como para os funcionários. Para isso, foi realizada a análise de atividades dos trabalhadores da cozinha, com ênfase na postura dos mesmos, com o auxílio de ferramentas ergonômicas que compreendem as reais condições que o operador está exposto, através do método OWAS (Ovako Working Posture Analysing System) e RULA (Rapid Upper Limb Assessment), utilizando o software Ergolândia 7.0. Para análise do ambiente, tomou-se como padrão o Manual da Associação Brasileira das Empresas de Refeições Coletivas (ABERC) de Práticas de Elaboração e Serviço de Refeições para Coletividade (2015). Os resultados mostraram que, para todos os funcionários analisados, e tomando como padrão o método RULA, deve ser realizada investigação na postura e ser introduzido mudanças para que torne melhor adaptado o ambiente de trabalho sem comprometer a postura corporal dos mesmos. Em relação ao ambiente, o restaurante é adaptado às normas estipuladas pela ABERC.

Palavras-chave: Ergonomia. OWAS. RULA. Alimentação. Ambiente.

\section{Introdução}

A ergonomia e as cozinhas industriais/unidades de alimentação têm uma relação mais estreita do que possa parecer em uma primeira análise. $\mathrm{O}$ estudo do tipo de trabalho executado e as condições nas quais estes trabalhos são executados podem ser úteis em várias etapas do ciclo de vida da unidade, trazendo benefícios tanto para os que trabalham dentro como os que se utilizam deste local.

Para Kroemer [1], o objetivo da ergonomia é criar empregos, sistemas ou produtos seguros, eficazes, eficientes, saudáveis, satisfatórios, convenientes e até agradáveis para o ser humano. Já para Wisner [2], a ergonomia baseia-se em conhecimentos no campo das ciências do homem (antropometria, fisiologia, psicologia, economia) com seus resultados traduzidos no dispositivo técnico (arte da engenharia ou arquitetura).
A ergonomia é diferenciada das outras áreas de conhecimento por sua característica interdisciplinar e pela sua natureza aplicada, ou seja, a adaptação do posto de trabalho e ambiente às características e necessidades do trabalhador [3].

Os produtos e postos de trabalho devem estar sempre adequados a população usuária, já que, de acordo com Fonseca [4] e Barbosa; Almeida [5], são muito comuns lesões reversíveis ou não, além de dor, fadiga, cansaço, indisposição e estresse decorrentes do intenso esforço físico e da permanência por muito tempo na mesma posição. Na maioria dos casos, estes problemas podem ser evitados através da melhoria dos postos de trabalho, objetos e mobiliários utilizados, e uma das formas de se obter esta melhoria é a sua adequação as diferenças corporais dos usuários.

Segundo a Norma Regulamentadora NR17, do Ministério do Trabalho e Emprego [6], as condições de trabalho incluem aspectos relacionados ao levantamento, transporte e descarga de materiais, ao mobiliário, aos equipamentos e às condições 
ambientais do posto de trabalho, e à própria organização do trabalho.

O processo de trabalho dentro de um restaurante exige que as atividades de preparo de alimentos, na maioria dos casos, sejam desenvolvidas em intenso ritmo e esforço por meio de movimentos repetitivos e monótonos, sob condições ambientais de ruído excessivo, temperatura elevada, iluminação precária, ventilação insatisfatória, além de serem utilizados equipamentos quebrados ou em condições arriscadas de funcionamento. Ainda é exigida a polivalência dos operadores, alocados a um número elevado de tarefas diferentes, de acordo com as necessidades do momento e a pressão gerada pelos horários em que as refeições devem ser servidas [7].

Ademais, a ergonomia apresenta-se como um custo benefício favorável, visto que ao investir em condições adequadas aos funcionários, evitam-se também afastamentos por motivo de doenças ocupacionais [8].

Devido a isso, este trabalho objetiva avaliar a postura corporal de funcionários e o ambiente em um restaurante situado no interior do Rio Grande do Sul.

\section{Metodologia}

O estudo foi realizado em um restaurante localizado no município de Tupanciretã/RS. Trata-se de uma empresa familiar, que possui 6 funcionários que trabalham alternadamente de acordo com a demanda. Além disso, trabalham os dois donos do local, sendo um deles o chef da cozinha e o outro responsável pelo caixa e pela parte administrativa.

O restaurante funciona ao meio dia de segunda à sábado, e à noite de sexta à domingo. Nos almoços é servido buffet a quilo e a noite a lá carte. A jornada de trabalho dos funcionários é, geralmente, em torno de $6 \mathrm{~h} / \mathrm{dia}$. Há rodízio de funcionários entre o dia e a noite nos finais de semana, para que não haja repetição das atividades 2 vezes no mesmo dia.

O estudo buscou realizar a análise e interpretação dos postos de trabalho de funcionários da cozinha do restaurante em estudo durante à noite. A cozinha é dividida em duas peças, uma para afazeres gerais e outra para alocação de fornos e chapas para cozinhar hambúrgueres.

Foram analisadas 3 atividades realizadas durante a confecção de hambúrgueres: 1 funcionário era responsável pela chapa (ação de cozinhar a carne), 1 funcionário cortava os pães e montava o hambúrguer e 1 funcionário picava os ingredientes e fritava algum alimento necessário.
Para a avaliação postural, foi realizado observações visuais dos funcionários durante a execução das tarefas e por registros fotográficos referentes às posições mais empregadas. Após, com o auxílio de ferramentas ergonômicas que compreendem as reais condições que o operador está exposto, utilizou-se os seguintes métodos: RULA (Rapid Upper Limb Assessment) e OWAS (Ovako Working Posture Analysing System), através da utilização do software Ergolândia 7.0 2019.

O método RULA consiste na avaliação da exposição dos trabalhadores a fatores de risco que podem ocasionar transtornos nos membros superiores do corpo [9] devido à sobrecarga biomecânica avaliando o posicionamento de braço, antebraço, punho, rotação do punho, pescoço, tronco e pernas, além de considerar a atividade a ser executada, o uso da musculatura para o desenvolvimento da atividade, bem como a carga necessária na operação. Por fim cada fator recebe uma determinada pontuação expressando o nível de intervenção necessária (Tabela 1).

Tabela 1 - Descrição da pontuação para cada nível de intervenção do método RULA

\begin{tabular}{ccc} 
Nível & Pontuação & Nível de intervenção \\
\hline 1 & 1 ou 2 & Postura aceitável \\
2 & 3 ou 4 & $\begin{array}{c}\text { Deve-se realizar uma observação. Podem ser } \\
\text { necessárias mudanças }\end{array}$ \\
3 & 5 ou 6 & $\begin{array}{c}\text { Deve-se realizar uma investigação. Devem ser } \\
\text { introduzidas mudanças }\end{array}$ \\
4 & 7 & $\begin{array}{c}\text { Podem ser introduzidas mudanças } \\
\text { imediatamente. }\end{array}$ \\
\hline
\end{tabular}

O método OWAS analisa as posturas das costas, braços, pernas e do esforço do trabalhador e também a questão do tempo que o trabalhador fica em cada postura, gerando, assim, códigos que são analisados (Tabela 2).

Tabela 2 - Descrição das categorias de ação (nível de ação) do método OWAS

\begin{tabular}{cc}
\hline Categorias & Nível de intervenção \\
\hline 1 & Não são necessárias medidas corretivas \\
2 & São necessárias correções em um futuro próximo \\
3 & São necessárias correções tão logo quanto possível \\
4 & São necessárias correções imediatas \\
\hline
\end{tabular}

Além da avaliação da postura corporal dos funcionários, foi realizado uma breve análise do ambiente, considerando as dimensões estruturais, os equipamentos e a higiene pessoal e do ambiente. Foi tomado como padrão o Manual da Associação 
Brasileira das Empresas de Refeições Coletivas (ABERC) de Práticas de Elaboração e Serviço de Refeições para Coletividade [10]. A utilização do mesmo justifica-se por este dispor sobre Boas Práticas de controle de processo de garantia de qualidade de produção de alimentos e da excelência dos serviços.

\section{Resultados e discussões}

A cozinha do referido restaurante tem área de circulação proporcionalmente boa para o número de funcionários. Além disso, consta de todos os equipamentos necessários para as atividades requeridas. Possui ar condicionado, iluminação boa, poucos ruídos e todos os funcionários utilizam os equipamentos de proteção e higiene (avental, luvas e toca para os cabelos). As ferramentas e utensílios são todos em bom estado e com limpeza adequada.

A jornada de trabalho dos funcionários é, aproximadamente, 36h/semanais, estando abaixo na média de estudos que apontam que, de acordo com Torres [11], é comum a carga horária de trabalho ser superior às 40 horas semanais, o que pode se tornar prejudicial à produção/hora, como ainda trazer um aumento do absenteísmo, acompanhado de doenças e acidentes.

$\mathrm{Na}$ (Tabela 3) está descrito alguns parâmetros de condições estruturais, de equipamentos e de higiene, verificando a conformidade do restaurante em relação ao Manual da ABERC [10].

Tabela 3 - Análise das condições estruturais, de equipamentos e de higiene do restaurante em relação às fornecidas pelo Manual da ABERC [10]

\begin{tabular}{cc}
\hline Condições Estruturais/Equipamentos/Higiene \\
\hline ABERC (2015) & Restaurante \\
\hline Áreas externas & $\mathrm{V}$ \\
Iluminação & $\mathrm{v}$ \\
Ventilação/Temperatura & $\mathrm{V}$ \\
Forros/Tetos & $\mathrm{x}$ \\
Cor & $\mathrm{V}$ \\
Sonorização & $\mathrm{v}$ \\
Piso & $\mathrm{V}$ \\
Paredes & $\mathrm{V}$ \\
Portas & $\mathrm{V}$ \\
Janelas & $\mathrm{v}$ \\
Móveis & $\mathrm{v}$ \\
Utensílios & $\mathrm{v}$ \\
Higiene Pessoal & $\mathrm{v}$ \\
Higiene do Ambiente & $\mathrm{v}$
\end{tabular}

De acordo com o Manual ABERC [10] a altura do pé direito em andares superiores deve ser de $2,7 \mathrm{~m}$ e o restaurante possui, em uma das cozinhas, 2,5m. Como a diferença não é significativa para um bom andamento do trabalho dos funcionários e ela ocorre somente em uma das cozinhas, pode-se dizer que o referido restaurante está em conformidade com o Manual ABERC [10].

A qualidade das refeições está diretamente relacionada ao desempenho da mão-de-obra [7]. De tal maneira, as condições físicas e ambientais das unidades de alimentação devem ser adequadas para que o trabalhador do setor de produção de refeições consiga manter a qualidade do produto e do serviço oferecido.

Além disso, segundo Siqueira et al. [12], os itens que podem impactar na qualidade de vida e na saúde do funcionário, bem como na sua produtividade, referem-se ao número de operadores e às atividades que são realizadas por cada um, jornada extensa, movimentos repetitivos, espaço físico inadequado, excesso de ruídos e temperatura elevada.

$\mathrm{Na}$ (Tabela 4) são apresentados os resultados obtidos pela metodologia OWAS de acordo com cada atividade: 1 - funcionário responsável pela chapa, 2 - funcionário responsável por cortar os pães e montar os hambúrgueres e 3 - funcionário responsável por picar e fritar alimentos.

Tabela 4 - Pontuação, nível de ação e intervenção para cada atividade realizada de acordo com a metodologia OWAS

\begin{tabular}{|c|c|c|c|c|}
\hline \multirow[b]{2}{*}{ Atividade } & \multicolumn{4}{|c|}{ Método OWAS } \\
\hline & $\begin{array}{c}\text { Postura } \\
\text { das } \\
\text { costas }\end{array}$ & $\begin{array}{c}\text { Postura } \\
\text { dos braços }\end{array}$ & $\begin{array}{c}\text { Postura das } \\
\text { pernas }\end{array}$ & $\begin{array}{c}\text { Categoria } \\
\text { de ação }\end{array}$ \\
\hline 1 & Ereta & $\begin{array}{c}\text { Um braço } \\
\text { no nível ou } \\
\text { acima dos } \\
\text { ombros }\end{array}$ & $\begin{array}{c}\text { De pé com } \\
\text { ambas as } \\
\text { pernas } \\
\text { esticadas }\end{array}$ & $\begin{array}{c}\text { Não são } \\
\text { necessárias } \\
\text { medidas } \\
\text { corretivas }\end{array}$ \\
\hline 2 & Inclinada & $\begin{array}{c}\text { Os dois } \\
\text { braços } \\
\text { abaixo dos } \\
\text { ombros }\end{array}$ & $\begin{array}{c}\text { De pé com } \\
\text { ambas as } \\
\text { pernas } \\
\text { esticadas }\end{array}$ & $\begin{array}{c}\text { São } \\
\text { necessárias } \\
\text { correções em } \\
\text { um futuro } \\
\text { próximo }\end{array}$ \\
\hline 3 & Ereta & $\begin{array}{c}\text { Os dois } \\
\text { braços } \\
\text { abaixo dos } \\
\text { ombros }\end{array}$ & $\begin{array}{c}\text { De pé com } \\
\text { ambas as } \\
\text { pernas } \\
\text { esticadas }\end{array}$ & $\begin{array}{c}\text { Não são } \\
\text { necessárias } \\
\text { medidas } \\
\text { corretivas }\end{array}$ \\
\hline
\end{tabular}

De acordo com a (Tabela 4), pode-se observar que a atividade 2 necessita de correções em um futuro próximo. Isso ocorre, possivelmente pelo fato de o funcionário responsável por essa atividade ter uma altura elevada, em torno de $190 \mathrm{~cm}$, ficando, dessa forma, com a coluna inclinada. 
Lopes e Oliveira [13] relatam a necessidade de uma maior orientação dos trabalhadores a respeito da manutenção da coluna de forma ereta e o flexionamento dos joelhos durante a realização das atividades, desta forma, concentrando os esforços nas pernas e evitando a sobrecarga na coluna lombar.

Uma alternativa para que esse funcionário não tenha problemas de coluna em um futuro próximo seria a adequação da altura da bancada em que realiza a atividade de cortar os pães e montar os hambúrgueres.

$\mathrm{Na}$ (Tabela 5) está apresentada os resultados obtidos pela metodologia RULA de acordo com cada atividade: 1 - funcionário responsável pela chapa, 2 - funcionário responsável por cortar os pães e montar os hambúrgueres e 3 - funcionário responsável por picar e fritar alimentos.

Tabela 5 - Pontuação, nível de ação e intervenção para cada atividade realizada de acordo com a metodologia RULA

\begin{tabular}{lccc}
\hline \multirow{2}{*}{ Atividade } & \multicolumn{3}{c}{ Método RULA } \\
\cline { 2 - 4 } & Pontuação & $\begin{array}{c}\text { Nível de } \\
\text { Ação }\end{array}$ & Intervenção \\
\hline 1 & 5 & 3 & $\begin{array}{l}\text { Deve-se realizar uma investigação. } \\
\text { Devem ser introduzidas mudanças }\end{array}$ \\
2 & 5 & 3 & $\begin{array}{l}\text { Deve-se realizar uma investigação. } \\
\text { Devem ser introduzidas mudanças } \\
\text { Deve-se realizar uma investigação. } \\
\text { Devem ser introduzidas mudanças }\end{array}$ \\
\hline
\end{tabular}

Nota-se, de acordo com a (Tabela 5), que para ambas as atividades há necessidade de correções tão logo quanto possíveis, o que contrapõe o método OWAS para as atividades 1 e 3 .

Uma possível intervenção para a atividade 1 seria adequar a altura da chapa para adequar melhor a postura dos braços do funcionário, que, no caso, estão mais abaixo que o nível horizontal da chapa.

Para a atividade 3 deve-se elevar a altura da bancada em que se realiza os cortes de alimentos, para que a coluna, braços e pescoço fiquem em um ângulo mais adequado.

De acordo com Vergara et al. [14], a ocorrência de fadigas musculares está ligada ao sobre carregamento muscular que permanece localizada em um determinado músculo. E, ainda, causam uma redução de desempenho do músculo fadigado, não apenas pela redução da força, mas também pela redução da velocidade de movimento. Isso explica os problemas de coordenação e aumento de erros e acidentes.
Como trata-se de uma cozinha, na qual possui utensílios pontiagudos (como facas) e produtos com elevada temperatura (óleo quente para fritura) há elevado risco de acidentes caso o funcionário não esteja ergonomicamente adaptado.

Além disso, pode-se influir que há necessidade de realizar análises em mais de uma metodologia, pois as mesmas podem apresentar resultados divergentes. A metodologia RULA abrange um maior número de parâmetros para análise, tornando, nesse estudo, a base para interpretação dos resultados.

\section{Conclusões}

A análise ergonômica do trabalho de funcionários de um restaurante no interior do Rio Grande do Sul revelou que, em relação ao ambiente, o mesmo está em conformidade com as normas da ABERC, e, em relação à postura corporal, devem ser tomadas providências para que a mesma não seja prejudicada. De tal forma, para um melhor desempenho é essencial que sejam impostas medidas em relação à altura dos móveis da cozinha, através da elevação e do rebaixamento dos mesmos.

Ademais, estando a qualidade das refeições diretamente relacionada ao desempenho da mão de obra é perceptível que são essenciais condições de trabalho satisfatórias, haja vista que um trabalho bem desenvolvido gera um produto final bem aceito.

\section{ERGONOMIC ANALYSIS OF WORK IN A RESTAURANT SITUATED INSIDE RIO GRANDE DO SUL}

ABSTRACT: Ergonomics differs from other areas of knowledge by its interdisciplinary nature and its applied nature, that is, the adaptation of the workplace and environment to the characteristics and needs of the worker. The objective of this study is to evaluate the environment and the body posture of employees of a private restaurant in the interior of Rio Grande do Sul. For this purpose, an analysis of the activities of the kitchen staff was carried out, with emphasis on the posture of the same, with the aid of ergonomic tools that comprise the actual conditions that the operator is exposed, using OWAS (Ovako Working posture analysis system) and RULA (Rapid Upper Limb Assessment), using software Ergolândia 7.0. For the analysis of the environment, the Manual of the Brazilian Association of Collective Meals Companies (ABERC) for the Elaboration and Service of Meals for Collectivity (2015) was adopted as standard. The results showed that, for all employees analyzed, and using the RULA method, research should be carried out and changes 
introduced to better adapt the work environment without compromising their body posture. In relation to the environment, the restaurant is adapted to the standards stipulated by ABERC.

Keywords: Ergonomics. OWAS. RULA. Food. Environment.

\section{Referências}

[1] KROEMER, K. H. E. Fitting the human: Introduction to ergonomic/human factors engineering (7th ed.). Boca Raton, FL: CRC Press, 2017.

[2] WISNER, A. Por dentro do trabalho: ergonomia: método \& técnica. Ftd. 1987.

[3] DUL, J.; WEERDMEESTER, B. Ergonomia prática. São Paulo: Edgard Blücher, 1995.

[4] FONSECA, P. S. Utilização da análise ergonômica do trabalho, enquanto uma ferramenta da ergonomia para a melhoria da qualidade do trabalho no setor de alimentação coletiva. IV Workshop de Análise Ergonômica do Trabalho; Viçosa. Minas Gerais: Universidade Federal de Viçosa, p.1-11, 2009.

[5] BARBOSA, L. N.; ALMEIDA, F. Q. A. Relato de experiência sobre a avaliação dos riscos ambientais e mapeamento em uma unidade de alimentação e nutrição (UAN) para a promoção da segurança no trabalho. Revista Simbio-Logias, Vol. 1, n. 2, p. 1-10, 2008.

[6] NORMA REGULAMENTADORA N 17 (NR-17) - Ergonomia. Ministério do Trabalho e Emprego. Disponível em: < http: www.mte.com.br >. Acesso em 13/06/2019.

[7] MATOS, C. H.; PROENÇA, R. P. C. Condições de trabalho e estado nutricional de operadores do setor de alimentação coletiva: um estudo de caso. Rev. Nutr. Vol. 16, n. 4, p. 493-502, 2003.

[8] CHOOBINEH, A. et al. Ergonomic intervention in carpet mending operation Applied Ergonomics, Vol. 35, n. 5, p. 493-496, 2004.

[9] KUMAR, A.; KAMATH, S. Avaliação rápida de membros superiores (RULA): evidências de validade e confiabilidade na identificação da ergonomia do local de trabalho entre funcionários de bancos que usam computadores. Revista Pesquisa em Fisioterapia, v. 9, n. 2, p. 194-203, 2019.

[10] ASSOCIAÇÃO BRASILEIRA DAS EMPRESAS DE REFEIÇÕES COLETIVAS (ABERC). Manual ABERC de práticas de elaboração e serviço de refeições para coletividades, São Paulo, Ed.11, p. 256, 2015.

[11] TORRES, M. G. V. Segurança no trabalho em Unidades de Alimentação e Nutrição - Treinamento e Dinâmicas. Brasília: Universidade de Brasília, 2003.
[12] SIQUEIRA, M. N. et al. Análise ergonômica do trabalho na churrascaria gaúcha. XXXI Encontro Nacional de Engenharia de Produção. Cenário Econômico Mundial Belo Horizonte, MG, Brasil, 04 a 07 de outubro de 2011.

[13] LOPES, E. S.; OLIVEIRA, F. M. Avaliação da carga de trabalho físico e da postura de trabalhadores na produção de mudas florestais. Cerne, Vol. 17, n. 4 , 2011.

[14] VERGARA, L. G. L. et al. Análise Ergonômica da atividade de Jardinagem e Paisagismo. Produção em Foco, Vol. 2, n. 1, 2012. 